\title{
Comportamiento del banco de semilla de malezas en un sistema agrícola-ganadero intervenido por diferentes tipos de labranzas
}

\author{
Behavior of the weed seed bank in an agricultural-livestock system intervened \\ by different types of tillage
}

\author{
Emiliano Foresto ${ }^{1}$ \\ Maria Andrea Amuchástegui ${ }^{2}$ \\ Cesar Omar Nuñez ${ }^{3}$ \\ Edgardo Zorza 4
}

\section{Resumen}

La mayoría de las malezas que emergen en los campos agrícolas provienen del banco de semillas del suelo. Los cambios que en él se producen son de vital importancia para el control de las malezas y generan variaciones en magnitud y tiempo de emergencia. El objetivo de este trabajo fue caracterizar la composición y abundancia del banco de semillas del suelo en dos tipos de labranzas bajo una rotación ganadera. El área de estudio está localizada en el establecimiento "Pozo del Carril", campo experimental de la Facultad de Agronomía y Veterinaria de la Universidad Nacional del Río Cuarto (F.A.V.-U.N.R.C.) cercano al paraje La Aguada, Argentina Se hicieron dos sistemas de labranza sin remoción (SR) y con remoción (CR). Se analizó el tamaño del banco de semillas, Riqueza (S), Diversidad específica $\left(\mathrm{H}^{\prime}\right)$ y Equidad ( $\left.\mathrm{J}^{\prime}\right)$, utilizando el método de lavado y tamizado. Se analizaron las profundidades: o-5 y 5-10 cm. El mayor tamaño del Banco de Semillas del Suelo se encontró en los primeros $5 \mathrm{~cm}$ del suelo para los dos tratamientos analizados y el valor más elevado lo mostró el tratamiento sin remoción en dicha profundidad, evidenciando cómo la remoción influye de manera diferencial sobre la distribución del banco de semillas de malezas en el suelo. Las especies anuales-estivales dominaron el banco de semillas en todos los tratamientos, siendo Eleusina indica la de mayor contribución. No se encontraron diferencias significativas entre riqueza, equidad y diversidad entre las labranzas analizadas. Se concluye que los sistemas de labranzas influyen en la distribución vertical de las semillas de malezas en los primeros $10 \mathrm{~cm}$ del suelo y afectan la composición y tamaño del banco de semillas de malezas.

\footnotetext{
1 Ingeniero Agrónomo. Docente de Botánica Sistemática Agrícola y Malezas. Universidad Nacional de Río Cuarto (UNRC), Río Cuarto, Córdoba, Argentina. eforesto@ayv.unrc.edu.ar Orcid: https://orcid.org/oooo-ooo2-8196-3030

2 Magister en Ciencia Agropecuarias. Docente de Botánica Sistemática Agrícola y Malezas. Universidad Nacional de Río Cuarto (UNRC), Río Cuarto, Córdoba, Argentina. aamuchastegui@ayv.unrc.edu.ar Orcid: https://orcid.org/oooo-ooo2-3682-6870

3 Magister en Botánica. Docente de Botánica Sistemática Agrícola y Malezas. Universidad Nacional de Río Cuarto (UNRC), Río Cuarto, Córdoba, Argentina. cnunez@ayv.unrc.edu.ar Orcid: https://orcid.org/oooo-ooo1-8744-3581

4 Ingeniero Agrónomo. Docente de Terapéutica Vegetal y Malezas. Universidad Nacional de Río Cuarto (UNRC), Río Cuarto, Córdoba, Argentina. ezorza@ayv.unrc.edu.ar Orcid: https://orcid.org/oooo-ooo2-1676-2742
}

Recibido: 08/01/2021 - Aprobado: 05/02/2021 
Palabras clave: banco de semillas; malezas; sistemas de labranza; pastura; siembra directa.

\section{Abstract}

Most of the weeds that emerge in agricultural fields come from the soil seed bank. The changes that occur in it are of vital importance for weed control and generate variations in magnitude and time of emergence. The objective of this work was to characterize the composition and abundance of the soil seed bank in two types of crops under a livestock rotation. The study area is located in "Pozo del Carril" establishment, an experimental field of the Agronomy and Veterinary Medicine Faculty at the National University of Río Cuarto (FAV-UNRC) near La Aguada, Argentina. removal (SR) and with removal (CR). The size of the seed bank, Wealth (S), Specific Diversity $\left(\mathrm{H}^{\prime}\right)$ and Equity $\left(\mathrm{J}^{\prime}\right)$ were analyzed, using the washing and sieving method. Depths were analyzed: 0-5 and 5-10 cm. The largest size of the Soil Seed Bank was found in the first $5 \mathrm{~cm}$ of the soil for the two treatments analyzed and the highest value was shown by the treatment without removal at that depth, showing how removal has a differential influence on the distribution of the soil and weed seed bank in the ground. Annual-summer species dominated the seed bank in all treatments, with Eleusina indicating the highest contribution. No significant differences were found between wealth, equity and diversity among the farms analyzed. It is concluded that tillage systems influence the vertical distribution of weed seeds in the first $10 \mathrm{~cm}$ of the soil and affect the composition and size of the weed seed bank.

Keywords: seed bank, weed, tillage systems, pasture, no-till.

\section{Introducción}

El objetivo de esta investigación es caracterizar la composición y tamaño del banco de semillas del suelo (BSS) en los primeros $10 \mathrm{~cm}$ de profundidad, en dos tipos de labranzas y una rotación agrícola-ganadero con el agregado de fertilizantes. El escrito se organiza en cuatro secciones. En primer lugar, se realiza una revisión bibliográfica. En segundo, se describen los aspectos metodológicos. En tercero, se discuten los principales resultados; finalmente, se exponen algunas conclusiones a las que pudimos arribar y se comenta cuáles serían los nuevos enfoques a brindarse en futuros estudios que aborden los bancos de semilla de malezas en suelos agrícola-ganaderos.

Gran parte de las malezas que emergen y logran completar su ciclo en los establecimientos agrícolas-ganaderos provienen del BSS. Los cambios que en él se producen juegan un papel crucial en el control de las malezas y generan variaciones en magnitud y tiempo de emergencia. Gran diversidad de trabajos han abordado el estudio del BSS en suelos agrícolas-ganaderos y pastizales naturales bajo diferentes tipos de laboreo (Leguizamón,1983), en relación con disturbios como el fuego o el pastoreo (Ernst 
y Morici, 2013) y la similitud entre la vegetación emergente y el BSS (Etchepare y Bocanelli, 2007), estudios en bosques nativos (Sione et al., 2015; Sabattini et al., 2018), pero son muy pocos los estudios relacionados a los sistemas agrícola-ganaderos que comparan sistemas de remoción de suelo y su relación con el comportamiento de las malezas en el BSS. Por lo expuesto, y debido a la importancia que tiene el BSS de malezas en los rendimientos de los cultivos ya que, son un reservorio importante de futuras nuevas plántulas se considera prioritario su estudio en este tipo de sistemas.

\section{Revisión de literatura}

El BSS se define como el conjunto de propágulos de semillas viables en el suelo y sobre su superficie en un área determinada, las que pueden persistir por períodos de tiempo largos o cortos (Haretche y Rodríguez, 2006). La mayoría de las malezas que crecen en los campos agrícolas provienen del BSS (Cavers, 1983). Los cambios en el banco de semillas del suelo son de vital importancia para el control de las malezas y en gran parte inciden en las variaciones en magnitud y tiempo de emergencia de las malezas (Mohler et al. 2006).

En los suelos cultivados, el banco de semillas está dominado, a menudo, por pocas especies de malezas, siendo estas especies de difícil control o aquellas más adaptadas a los sistemas de cultivo (Gomes y Christoffoleti, 2008). Alrededor del 60\% de las semillas totales de malezas se encuentra entre o y $5 \mathrm{~cm}$ de profundidad del suelo y la concentración de semillas de las mismas, disminuye logarítmicamente con la profundidad del suelo (Chauhan et al., 2011). Esta acumulación debería disminuir con la tecnología utilizada y la aplicación estratégica de herbicidas durante la producción de cultivos, debido a que muchas plántulas emergen todos los años y con los controles químicos aplicados se debería estar evitando una nueva lluvia de semillas al suelo, sin embargo, esto no sucede. Por lo tanto, desde el punto de vista del manejo, convendría prestar más atención en los controles al final del ciclo de los cultivos, ya que muchas malezas emergen tardíamente y alcanzan el estado reproductivo, incorporando semillas al banco. Junto con la labranza, otras prácticas como el mulching, la rotación de cultivos y la aplicación de herbicidas también afectan el destino de las malezas en el próximo cultivo (Swanton et al., 2008; Nichols et al., 2015).

La labranza induce cambios en el microclima del suelo, por ejemplo, en la humedad, la temperatura y la luz (Alvaro-Fuentes et al., 2007), dichos factores se ven modificados en el perfil del suelo por lo que, la posición de las semillas en el mismo es uno de los principales factores que influyen en la germinación y la emergencia de plántulas. En sistemas SR, por ejemplo, pueden incrementar la proporción de semillas retenidas sobre la superficie del suelo, comparado con el arado de rejas (Ghersa y Martínez Ghersa, 2000). Éste último, al invertir el pan de tierra, disminuye la germinación potencial de las semillas ubicadas sobre la superficie, al mismo tiempo que ubica las semillas enterradas en profundidad en superficie y les confiere las condiciones para 
germinar (Carter e Ivani, 2006).En los suelos no laboreados, debido a que las semillas se localizan en la capa superior del suelo y a su vez estas, se percolan en el suelo a través de procesos muy lentos (grietas, fauna, ciclos liofilizados), provoca que la emergencia sea superior al $80 \%$ en los primeros 2,5 cm del suelo (Albrecht y Auerswald, 2009).

\section{Materiales y métodos}

\section{Área de estudio}

El área de estudio está localizada en el Establecimiento "Pozo del Carril", campo experimental de la F. A. V. - U. N. R. C. cercano al paraje La Aguada, ubicado a $30 \mathrm{Km}$. al oeste de la ciudad de Río Cuarto, Córdoba, Argentina localidad ubicada a $32^{\circ} 51^{\prime} \mathrm{LS}$, $64^{\circ} 40^{\prime} \mathrm{LW}$ y 550 metros snm. El suelo es un Hapludol Típico de textura franco arenosa.

\section{Diseño experimental}

El diseño experimental utilizado fue completo aleatorizado. El BSS fue muestreado en Marzo del 2014, luego de la diseminación de las especies estivales, y previo a la germinación de las malezas invernales. Se trabajó sobre un ensayo de sistemas de labranzas iniciado en la campaña 1995/96, con una rotación agrícola (1995/99) de maíz-girasol; luego, pastura de alfalfa en mezcla con gramíneas forrajeras (1999/o2). Posteriormente, rotación agrícola de maíz-soja (2002-2007), pastura base alfalfa (2007-2010), agrícola de maíz-soja (2010-20013) y alfalfa pura, sembrada en el año 2014, realizada en plano a o,22 m entre líneas. Los cultivos fueron sembrados en plano a $0.70 \mathrm{~m}$ entre líneas. Además, el ensayo constó de tratamientos con el agregado de fertilizante, llevado a cabo en los dos sistemas de labranza y fue a base de fosfato di amónico (100 Kg/ha. a la siembra) y urea ( $120 \mathrm{Kg} / \mathrm{ha}$. en V6) en maíz y; superfosfato triple (100 Kg/ha.) y yeso (100 Kg/ha. a la siembra) en soja.

\section{Estos sistemas fueron conducidos en dos tratamientos:}

$>$ T1: Sin remoción de suelo (SR): Sin remoción del suelo y con aplicación de glifosato en pre-siembra.

$>$ T2: Con remoción de suelo (CR): en base a arado de rejas más rastra de discos de tiro excéntrico $(1995-2010)$ y $l$ en base a arado de cincel y rastra de discos de tiro excéntrico (2011-2014).

Para cada tratamiento ( $\mathrm{SR}$ y $\mathrm{CR}$ ) se tomaron 10 réplicas (muestras compuestas) de cinco submuestras de suelo (Figura 1). Para ello, se utilizó un cilindro de $3 \mathrm{~cm}$ de diámetro por $10 \mathrm{~cm}$ de longitud, separando en diferentes profundidades $(0-5 \mathrm{~cm}$ y $0-10 \mathrm{~cm})$ ). Cada profundidad fue muestreada en forma independiente. El tamaño total del BSS fue considerado de $\mathrm{o}$ a $10 \mathrm{~cm}$ de profundidad. 
Para la separación de las semillas se utilizó el método por lavado y tamizado; para lo cual, las muestras de suelo fueron colocadas en botella de 2.5 L., agregándole $750 \mathrm{~g}$. de sal y agua hasta el llenado de las mismas. Se agitaron fuertemente en forma manual y se dejaron reposar 48 hs. Luego se filtró el contenido en una serie de tamices de 10, 0.2 y o,02 mm de diámetro (Thompson et al., 1997) y posteriormente se lavó con agua varias veces para separar el suelo de las semillas. El producto del filtrado se colocó sobre papel de filtro y se secó en estufa a $40{ }^{\circ} \mathrm{C}$, durante $48 \mathrm{hs}$. Luego se procedió a la identificación y cuantificación de las semillas en un microscopio estereoscópico y referido a m2.

Para caracterizar el Banco de Semillas del Suelo (BSS) se calcularon los siguientes parámetros: Tamaño del banco, Riqueza $(S)$, Diversidad específica $\left(\mathrm{H}^{\prime}\right)$, Equidad $\left(\mathrm{J}^{\prime}\right)$, Similitud (QS).

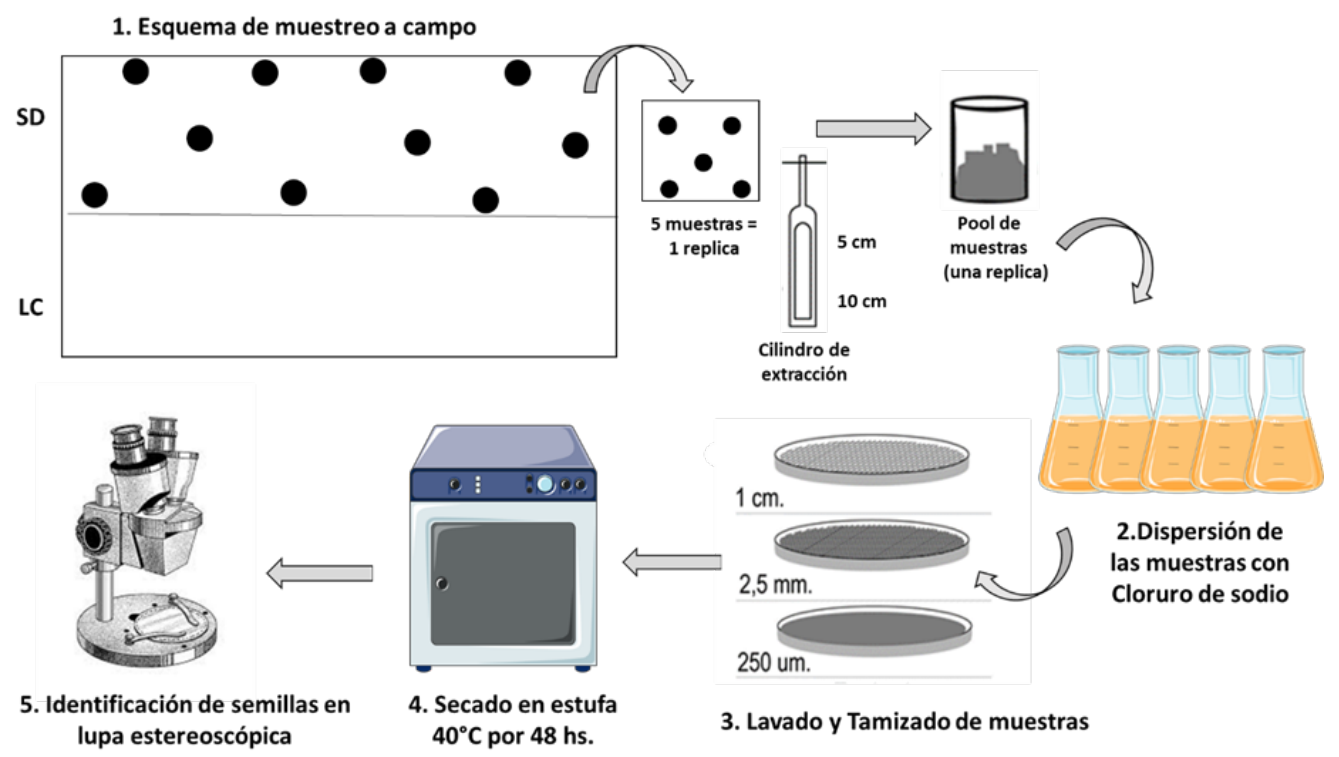

Figura no. 1. Detalle del diseño experimental y sistema de muestro empleado para la evaluación del BSS.

\section{Análisis estadístico}

Los datos se analizaron a través de un ANAVA de una o dos vías de acuerdo al caso y comparación de las medias con test Tukey, Prueba de Kruskal-Wallis con test de rangos. Se analizó la riqueza, y la diversidad específica a través del índice de ShannonWeaver. El análisis estadístico se ejecutó con el programa InfoStat (Di Rienzo et al. 2018). Para los test estadísticos se utilizó el nivel de significancia de ( $<<0,05)$. 


\section{Resultados y discusión}

\section{Análisis de la diversidad del BSS de malezas}

A partir del muestreo realizado se observó que el BBS estuvo constituido por 25 especies, las cuales pertenecen a 17 familias. Siete, pertenecen al grupo de las monocotiledóneas y los 18 restantes a las dicotiledóneas, resultados similares fueron descriptos por Puricelli y Tuesca (2005), y en particular en SR la proporción de especies monocotiledóneas fue menor que en CR. Del total de especies, seis fueron anuales invernales, nueve anuales estivales, seis perennes estivales y cuatro perennes invernales (Tabla 1.).

Tabla no. 1: Descripción de las especies de malezas en el banco de semillas del suelo $(0-10 \mathrm{~cm}$ de profundidad). Los $(*)$ indican especies identificadas como resistentes al glifosato según SENASA, 2020. Los nombres científicos fueron actualizados de acuerdo a la página web del Instituto de Botánica Darwinion (2019):http://buscador.floraargentina.edu.ar/ (02/02/2021)

\begin{tabular}{|c|c|c|c|c|}
\hline Nombre cientifico & Nombre vulgar & Familia & Ciclo biológico & $\begin{array}{l}\text { Ciclo de } \\
\text { crecimiento }\end{array}$ \\
\hline $\begin{array}{l}\text { Amaranthus hybridus L. subsp. } \\
\text { hybridus* }\end{array}$ & Yuyo colorado & Amaranthaceae & Anual & Estival \\
\hline Ammi majus L. & Falsa viznaga & Apiaceae & Anual & Invernal \\
\hline Ammi visnaga (L.) Lam. & Viznaga & Apiaceae & Anual & Estival \\
\hline Anoda cristata (L.) Schltdl. & Oreja de gato & Malvaceae & Anual & Estival \\
\hline Brassica rapa L. ${ }^{*}$ & Nabo & Brassicaceae & Anual & Invernal \\
\hline $\begin{array}{l}\text { Buglossoides arvensis (L.) I.M. } \\
\text { Johnst. }\end{array}$ & Yuyo moro & Boraginaceae & Anual & Invernal \\
\hline Chenopodium album L. & Quínoa & Chenopodiaceaeee & Anual & Estival \\
\hline Convolvulus arvensis L. & Correhuela & Convolvulaceae & $\begin{array}{l}\text { Perenne } \\
\text { erenne }\end{array}$ & Invernal \\
\hline Cyperus rotundus L. & Cebollín & Cyperaceae & Perenne & Estival \\
\hline $\begin{array}{l}\text { Descurainia erodiifolia (Phil.) } \\
\text { Prantl ex Reiche }\end{array}$ & $\begin{array}{l}\text { Altamisa colorada } \\
\text { colorada }\end{array}$ & Brassicaceae & Anual & Invernal \\
\hline Digitaria sanguinalis (L.) Scop. & Pata de gallina & Poaceae & Anual & Estival \\
\hline Echinochloa crus-galli (L.) & Capín & Poaceae & Anual & Estival \\
\hline Eleusine indica (L.) Gaertn.* & Pie de gallina & Poaceae & Anual & Estival \\
\hline Mollugo verticillata $L$. & Mollugo & Molluginaceae & Anual & Estival \\
\hline Oxalis conorrhiza Jaca. & Vinagrillo & Oxalidaceae & Perenne & Invernal \\
\hline Panicum bergii Arechav. & Paja voladora & Poaceae & Perenne & Estival \\
\hline Polygonum aviculare L. & Cien nudos & Polygonaceae & Anual & Invernal \\
\hline
\end{tabular}


Comportamiento del banco de semilla de malezas en un sistema agrícola-ganadero intervenido por diferentes tipos de labranzas

\begin{tabular}{|l|l|l|l|l|}
\hline \multicolumn{1}{|c|}{ Nombre cientifico } & \multicolumn{1}{|c|}{ Nombre vulgar } & \multicolumn{1}{c|}{ Familia } & Ciclo biológico & \multicolumn{1}{c|}{$\begin{array}{c}\text { Ciclo de } \\
\text { crecimiento }\end{array}$} \\
\hline Portulaca oleracea L. & Verdolaga & Portulacaceae & Anual & Estival \\
\hline Raphanus sativus L.* & Nabón & Brassicaceae & Anual & Invernal \\
\hline Rumex crispus L. & Lengua de vaca & Polygonaceae & Perenne & Invernal \\
\hline $\begin{array}{l}\text { Setaria parviflora (Poir.) } \\
\text { Kerguélen. }\end{array}$ & Baraval & Poaceae & Perenne & Estival \\
\hline Setaria viridis (L.) P. Beauv. & Cola de zorro & Poaceae & Anual & Estival \\
\hline Sonchus oleraceus L. & Cerraja & Asteraceae & Perenne & Estival \\
\hline Sorghum halepense (L.) Pers.* & Sorgo de alepo & Poaceae & Perenne & Estival \\
\hline Verbena bonariensis L. & Verbena & Verbenaceae & Perenne & Invernal \\
\hline
\end{tabular}

Con relación al ciclo de crecimiento, predominaron las especies primavero-estivales en ambos tratamientos, obteniendo SR el menor porcentaje. Este resultado podría indicar que los programas de control químicos de malezas aplicados durante la rotación no han sido totalmente efectivos debido a que, el predominio de cultivos estivales sembrados debería aumentar la presión de control sobre este grupo de malezas, las que se esperaría que tengan menor participación en el BSS total. Esto podría tener su origen con los escapes de malezas hacia finales de ciclo de los cultivos los cuales no reciben ningún control químico ya que se considera que su influencia es baja en los rendimientos esperados favoreciendo que las malezas cumplan su ciclo en el lote, llegando a fructificar y dispersar sus semillas.

La proporción de malezas perennes fue menor al 2\% en las dos labranzas, lo cual es importante de remarcar ya que las mismas son un problema cuando el objetivo es la producción forrajera en este caso alfalfa, especialmente luego de 2 a 3 años de reducción o ausencia de laboreo, obligando a los productores a optar por otras alternativas de manejo.

La reducción de monocotiledóneas anuales en el sistema SR, en comparación con $\mathrm{CR}$, puede atribuirse a la menor tasa de germinación por las menores temperaturas que ocurren en sistemas sin remoción de suelo. Además, la mayor cantidad de residuos en superficie reducen la transmisión de la luz hacia el suelo (Chauhan y Johnson, 2011). Otras posibles causas son la reducción de artrópodos en los sistemas SR que se alimentan selectivamente de semillas de malezas latifoliadas y en menor grado de gramíneas anuales. De esta forma se han constituido en uno de los principales problemas para los productores pampeanos que adoptan sistemas de labranzas conservacionistas.Las latifoliadas anuales tuvieron un mayor valor en la contribución porcentual del BSS en $\mathrm{SR}$, ya que son, en general, favorecidas por los sistemas conservacionistas (Puricelli y Tuesca, 2005). 
En los dos tratamientos y dos profundidades analizadas predominan Amaranthus hybridus, Chenopodium album, Eleusine indica, Digitaria sanguinalis y Polygonum aviculare. Las cuatro primeras son de ciclo primavero-estival lo que podría reflejar el predominio de cultivos estivales en el último tramo de la historia de uso del lote, con posterioridad al inicio del ciclo de pastura. Eleusine indica la especie de mayor contribución porcentual al banco de semillas del suelo; contribuyo en CR con más de 60\% al total del BSS.

La alta infestación de gramíneas anuales en esta pastura, puede deberse a que adaptan su ciclo de crecimiento al cultivo implantado y con sucesivas cohortes de nacimiento a lo largo del ciclo del cultivo dado que éste, a lo largo de su ciclo ontogénico, atraviesa diferentes estadios fenológicos que actúan como filtros en la estructuración de las comunidades de malezas. El conopeo del cultivo que acompaña generalmente a la comunidad de malezas modifica los factores térmicos y lumínicos en el microambiente de las semillas de manera tal que algunas semillas que requieran de la terminación de la dormición no vean satisfecha esa demanda (Guglielmini, 2015).

La mayor riqueza de especies en SR, entre los o-5 cm profundidad de suelo, podría deberse a la acumulación de las semillas de diferentes especies que puedan crecer en ese ambiente. En cuanto al índice de equidad bajo en todos los tratamientos podría deberse al gran aporte que realiza Eleusine indica siendo una especie que no es apetecible por el ganado y por lo tanto de continuar esta pastura se incrementaría aún más esta especie de maleza.

La similitud florística en el estrato de 5 a $10 \mathrm{~cm}$ es mayor entre SR, y podría deberse a que el tipo de labranza no producen disturbios importantes a esta profundidad. La riqueza de especies en $C R$, en la profundidad de $0-5 \mathrm{~cm}$, no se diferenció estadísticamente de la profundidad de 5-10 cm; y esto se puede explicar debido a la inversión del pan de tierra que se produce en el momento del laboreo del suelo y que permite la mezcla de semillas que estaban en la superficie con aquellas que ya se encontraban enterradas a causa de laboreos anteriores. La diversidad específica y la equidad son menores en el primer estrato del perfil y tienden a aumentar con la profundidad del suelo, encontrando resultados opuestos a los de Amuchástegui (2012). En SR la riqueza es máxima en $0-5 \mathrm{~cm}$ de suelo disminuyendo al llegar a 5-10 $\mathrm{cm}$ de profundidad y se reduce el disturbio en el perfil. La diversidad específica no tiene diferencias significativas. La similitud florística en el BSS en general fue baja y en ambas profundidades la similitud fue mayor en $C R$ lo cual podría explicarse por el grado de remoción al que el perfil de suelo se somete.

\section{Análisis del Tamaño del BSS}

Cuando se comparó el tamaño total del BBS de malezas entre los tratamientos, no se registró diferencia estadísticamente significativa, siendo en CR levemente mayor en valor absoluto ( $15780 \pm 1874)$, mientras que SR fue de (15322 \pm 1581 ) (Figura 2). Estos 
resultados concuerdan en parte con lo expresado por Lutmam et al. (2002) afirma que, los sistemas SR reducen el disturbio del suelo e incrementan la proporción de semillas cerca de la superficie del mismo.

Los antecedentes revisados indican que en los sistemas SR debería tener mayor tamaño de BSS (Ghersa y Martínez Ghersa, 200o); sin embargo, en este estudio el sistema $C R$ fue realizado con un cincel y luego una rastra de tiro excéntrico, lo cual no invierte el pan de tierra, de allí es que entre ambos tratamientos no se encontraron diferencias en el tamaño de BSS total (o-10 cm). Entre los 0-5 $\mathrm{cm}$ de profundidad los mayores tamaños del BSS se presentaron en SR, no presentando diferencias significativas con el sistema $C R$. En SR se incrementa la proporción de semillas retenidas en la superficie del suelo . Ello se debe a que, en los sistemas SR, la lluvia de semillas de malezas se deposita principalmente en la superficie del suelo. En la profundidad 5-10 cm no se manifestaron diferencias significativas en los tamaños de los BSS entre las labranzas, esto se debería a que es una profundidad de transición de los tamaños de estos bancos en los distintos tratamientos, donde a medida que se incrementa la profundidad disminuye la cantidad de semillas por unidad de superficie.

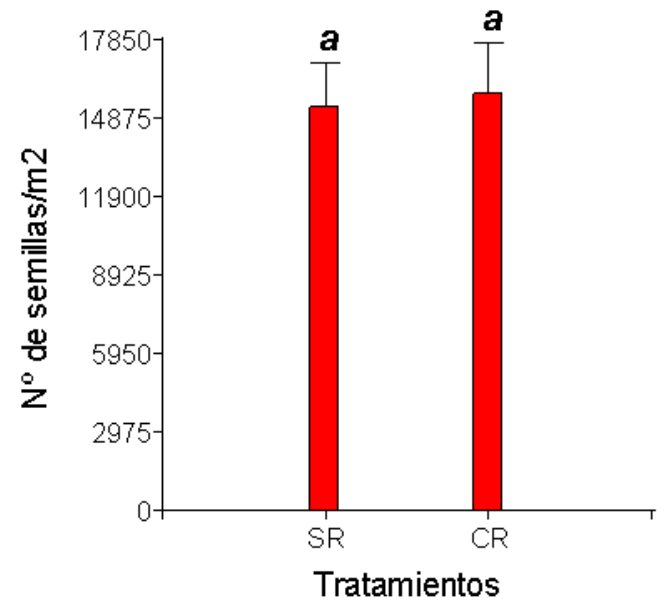

Figura no. 2. Tamaño total del BSS de los diferentes sistemas de labranzas de 0 a $10 \mathrm{~cm}$ de profundidad. SR: Sin Remoción y CR: Con remoción. Letras distintas indican diferencias significativas $(p<0,05)$.

Observamos que a medida que aumenta la profundidad, el tamaño del BSS disminuye en CR. Lo que podría ser explicado por la labranza, ya que, al invertir el pan de tierra, se disminuye la germinación potencial de las semillas ubicadas sobre la superficie y al mismo tiempo transfiere las semillas enterradas en profundidad hacia la superficie y de esta manera les confiere las condiciones para germinar (Carter e Ivani, 2006). 
En la tabla 2 se observaron las diferencias para riqueza, equidad y diversidad entre los distintos tipos de tratamientos teniendo en cuenta las diferentes profundidades de muestreo. Los resultados sugieren que no hubo diferencias estadísticamente significativas para riqueza y diversidad especifica entre las labranzas en las diferentes profundidades, siendo SR la que presento el mayor valor de riqueza y diversidad.

Tabla no. 2: Índices de riqueza (S), equidad (J') y de Shannon-Weaver $\left(H^{\prime}\right)$ en el BSS para todas las labranzas y profundidades analizadas. Letras distintas indican diferencias significativas $(p<0,05)$.

\begin{tabular}{|c|c|l|l|c|}
\hline Profundidad (cm) & Labranza & \multicolumn{1}{|c|}{$\mathbf{S}$} & $\mathbf{J}^{\prime}$ & $\mathbf{H}^{\prime}$ \\
\hline \multirow{2}{*}{$0-5$} & $\mathrm{CR}$ & 9 & 0,33 & $0,74 \mathrm{a}$ \\
\cline { 2 - 5 } & $\mathrm{SR}$ & 14 & 0,58 & $1,53 \mathrm{~b}$ \\
\hline \multirow{2}{*}{$5-10$} & $\mathrm{CR}$ & 7 & 0,52 & $1,01 \mathrm{a}$ \\
\cline { 2 - 5 } & $\mathrm{SR}$ & 8 & 0,51 & $1,07 \mathrm{a}$ \\
\hline \multirow{2}{*}{$0-10$} & $\mathrm{CR}$ & 11 & 0,38 & $0,91 \mathrm{a}$ \\
\cline { 2 - 5 } & $\mathrm{SR}$ & 16 & 0,59 & $1,65 \mathrm{~b}$ \\
\hline
\end{tabular}

La diversidad específica es mayor en SR, no diferenciándose significativamente del resto de los tratamientos, debido a un mayor índice de equidad. Cuando se analizó la profundidad de $0-10 \mathrm{~cm}$ se vio que hubo diferencias estadísticamente significativas, siendo SR la que mostró mayores valores de diversidad y riqueza. En la menor profundidad ocurrió lo mismo que en el análisis anterior, SR obtuvo los mayores valores de diversidad y riqueza, mientras que en la profundidad de $5-10 \mathrm{~cm}$ no hubo diferencias significativas entre las labranzas.

\section{Comportamiento de las labranzas por profundidad de suelo con respecto al tamaño y composición del BSS}

Cuando se comparó los resultados obtenidos de número de semillas con respecto a la profundidad de estudio, no se observaron diferencias estadísticamente significativas entre ambas profundidades analizadas (figura 3). En la profundidad de $\mathrm{o}-5 \mathrm{~cm}$, la mayor cantidad de semillas fue registrada en SR con $12162 \pm 1430$; mientras que CR obtuvo $9917 \pm 2053$; sin presentar diferencias estadísticamente significativas. En la profundidad de $5-10 \mathrm{~cm}$, la mayor cantidad de semillas fue registrada en $\mathrm{CR}$ con $5863 \pm 1452$; mientras que en $S R 3160 \pm 521$ respectivamente; no existiendo diferencias significativas entre los dos tratamientos a esta profundidad.

En la figura 3 se observó que los mayores valores de tamaño del BSS lo presentaron ambos tratamientos en los primeros $5 \mathrm{~cm}$ de suelo, no habiendo diferencias estadísticamente significativas entre ambas, de igual manera se comportó la profundidad 
de 5-10 cm cuando se comparó entre ambos tratamientos. Sí se presentó diferencia significativa entre las profundidades en SR, obteniendo los mayores valores la profundidad de o-5 cm. En CR no hubo diferencias estadísticamente significativas entre ambas profundidades.

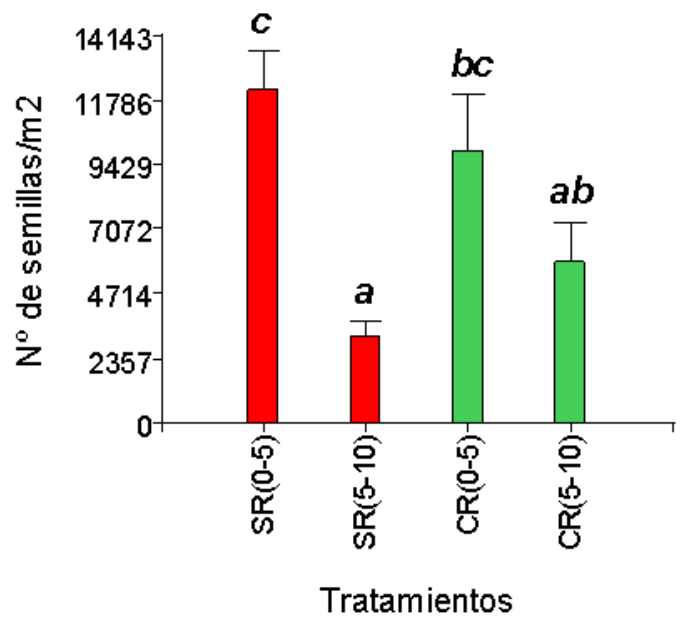

Figura no. 3. Tamaño total de BSS en las distintas profundidades según la presencia o ausencia de un sistema de remosion. SR: Sin Remoción y CR: Con remoción. Letras distintas indican diferencias significativas $(p<0,05)$

La especie que tuvo la mayor contribución al tamaño del BSS fue Eleusine indica en las labranzas, aunque la contribución porcentual fue diferente en cada tratamiento. En CR, fue de $73.52 \%$, mientras que en SR $35.17 \%$. Además de Eleusine indica, estuvieron presentes Digitaria sanguinalis, con una contribución de $21.47 \%$ en SR, mientras que en CR solo lo hizo en $1.84 \%$, Polygonum aviculare con un $12.52 \%$ CR y en SR con un $20.49 \%$ fueron especies cuya contribución porcentual fue mayor en SD que en LC. En CR solamente con dos especies se llega al 86 \% del total de la contribución al BSS, en tanto que en SR la contribución muestra mayor equidad (Tabla 3).

Tabla no. 3: Contribución porcentual de las nueve especies con mayor aporte al BSS de o-10 cm en los distintos sistemas de labranzas.

\begin{tabular}{|l|c|l|l|}
\hline \multicolumn{2}{|c|}{ SR } & \multicolumn{2}{c|}{ CR } \\
\hline \multicolumn{1}{|c|}{ Especies } & $\%$ & \multicolumn{1}{c|}{ Especies } & $\%$ \\
\hline Eleusine indica & 35.17 & Eleusine indica & 73.52 \\
\hline Digitaria sanguinalis & 21.47 & Polygonum aviculare & 12.52 \\
\hline Polygonum aviculare & 20.49 & Amaranthus hybridus & 9.88 \\
\hline Amaranthus hybridus & 15.13 & Digitaria sanguinalis & 1.84 \\
\hline Chenopodium album & 2.85 & Brassica rapa & 0.92 \\
\hline Ammi majus & 1,09 & Portulaca oleracea & 0.53 \\
\hline
\end{tabular}




\begin{tabular}{|l|c|l|l|}
\hline \multicolumn{2}{|c|}{ SR } & \multicolumn{2}{c|}{ CR } \\
\hline \multicolumn{1}{|c|}{ Especies } & $\%$ & \multicolumn{1}{c|}{ Especies } & $\%$ \\
\hline Setaria viridis & 0,68 & Chenopodium album & 0.26 \\
\hline Brassica rapa & 0,68 & Oxalis conorriza & 0.13 \\
\hline Echinochloa crusgalli & 0,27 & Sonchus oleraceus & 0.13 \\
\hline
\end{tabular}

\section{IV.Conclusiones}

Aunque los estudios sobre bancos de semillas de malezas no proporcionan información de las malezas sobre el suelo, son fundamentales para comprender y predecir la evolución de comunidades de malezas, ya que el banco de semillas refleja la historia en el campo. Los resultados sugieren que la rotación de cultivos y la técnica de labranza actúan como filtros que a menudo interactúan entre sí para determinar la composición y abundancia de especies de malezas en el banco de semillas del suelo. Por el contrario, el sistema de labranza no tuvo efecto en el tamaño del banco de semillas de malezas, pero modifico significativamente su composición, así como la distribución de semillas de malezas en el perfil del suelo. El mayor tamaño del BSS se encontró en los primeros $5 \mathrm{~cm}$ del suelo para los dos tratamientos analizados y el valor más elevado lo mostró SR en dicha profundidad, dejando en evidencia como la remoción influyen de manera diferencial sobre la distribución del banco de semillas de malezas en el suelo.

La adopción de un sistema $\mathrm{CR}$ (basada en arado de cincel y rastra de discos de tiro excéntrico) favoreció algunas especies de malezas (principalmente E. indica); mientras que, por su parte, el uso continuo de SR condujo a un aumento en semillas de malezas en la capa superior del suelo y un significativo aumento en la densidad de semillas de algunas especies problemáticas. Se encontraron 5 especies que presentan resistencia al glifosato; las cuales fueron: Eleusine indica, Amaranthus hybridus, Brassica rapa, Rhapanus sativus y Sorghum halepense. De estas especies, las 3 primeras contribuyen a formar el 85\% del BSS en el tratamiento CR y el 50\% en SR, evidenciando de esta manera una alta dominancia de malezas resistentes al glifosato en el total del BSS.

Estos resultados sugieren que, aunque los sistemas SR son amigable con el medio ambiente porque mitiga la erosión del suelo, reduce el uso de energía y mejora el hábitat de la vida silvestre, los agricultores sólo deben aplicar tal conservación del suelo técnicamente dentro de una secuencia de cultivo apropiada. En particular el continuo monocultivo de cultivos anuales estivales soja-maíz de resultó en un aumento en la densidad total del banco de semillas de malezas y, al mismo tiempo, una reducción en la diversidad de malezas, con un marcado aumento en algunas especies, algunas de las cuales son potencialmente difíciles de controlar. Estos resultados son preliminares, resulta pertinente seguir analizando el BSS transcurridas varias temporadas de lluvia de semillas. 


\section{Lista de referencia}

Albrecht, H., y Auerswald, K. (2009). Seed traits in arable weed seed banks and their relationship to land-use changes. Basic and Applied Ecology, 10(6), 516-524.

Álvaro-Fuentes, J., Cantero-Martínez, C., López, M. V., y Arrúe, J. L. (2007). Soil carbon dioxide fluxes following tillage in semiarid Mediterranean agroecosystems. Soil and Tillage Research, 96(1-2), 331-341.

Amuchástegui, M. A.; Nuñez, C. O.; Balzola, C.; Zorza, E. Y Mulko, J (2012). Distribución vertical de las semillas de malezas, bajo diferentes tipos de labranzas en un sistema de rotación agrícola. XIV Jornadas Fitosanitarias Argentinas/ compilado por Nora Raquel Andrada et al.- $1^{\text {a }}$ Ed.- Villa Mercedes. 10 pp. (cd-rom) ISBN 978-950-609-073-9

Carter, M. R. y J. A. Ivany. (2006). Weed seed bank composition under three longterm tillage regimes on a fine sandy loam in Atlantic Canada. Soil Tillage Res. 90: 29-38.

Cavers, P. B. (1983). Seed demography. Canadian journal of botany, 61(12), 3578-3590.

Chauhan, B. S. y D. E. Johnson 2011. Ecological studies on Echinochloa cruss-galli and the implications for weed management in direct-seeded rice. Crop Protection 30: 1385-1391.

Di rienzo J. A., F. Casanoves, M. G. Balzarini, L. Gonzalez, M. Tablada y C.W. Robledo. InfoStat versión (2018). Grupo InfoStat, FCA, Universidad Nacional de Córdoba, Argentina. URL http://www.infostat.com.ar

Ernst, R. D., y Morici, E. (2013). Germinable seed bank of grasses in the caldenforests before and after dispersal differences. Revista de la Facultad de Agronomía de la Universidad Nacional de La Pampa, 22(Suplemento 2 Tomo I), 39-44.

Etchepare, M. A., y Boccanelli, S. I. (2007). Análisis del banco de semillas y su relación con la vegetación emergente en una clausura de la llanura pampeana. Ecología austral, 17(1), 159-166.

Ghersa, C. M. y M. A. Martínez Ghersa. (2000). Ecological correlates of weed size and persistente in the soil under different tilling systems: implications for weed management. Field Crop Res. 67: 141-148.

Gomes FG, Christoffoleti PJ. Biology and weed management inno-tillage areas. Plant. Dan. 2008;26(4):789-798. English. 


\section{AGROPECUARIA}

Guglielmini, A. C., Kruk, B. C., \& Satorre, E. H. (2015). Analyzing weed community changes in extensive farming systems of the Pampas through a functional approach. Agronomía \& Ambiente, 35(2), 121-130.

Haretche F. y C. Rodríguez. (2006). Banco de semillas de un pastizal uruguayo bajo diferentes condiciones de pastoreo. Ecología Austral. 16: 105113.

Instituto de botánica darwinion (2019). Base de datos "Flora Argentina". Disponible en: http://www.floraargentina.edu.ar

Leguizamón, E. S. (1983). Población dinámica. el sorgo de Alepo en la secuencia trigo/soja. Desarrollo rural. EEA Famaillá. a. 3, (24).

Lutman, P. J. W., Cussans, G. W., Wright, K. J., Wilson, B. J., Wright, G. Mc. N. y Lawson. H. M. (2002). The persistence of seeds of 16 weeds species over six years in two arable fields. Weed Res. 42, 231-241.

Mohler, C. L., Frisch, J. C. y C. E. Mc. Culloch. (2006). Vertical movement of weed surrogates by tillage implements and natural processes. Soil \& Tillage Res. 86, 110-122.

Nichols, V., Verhulst, N., Cox, R., y Govaerts, B. (2015). Weed dynamics and conservation agriculture principles: A review. Field Crops Research, 183, 56-68.

Puricelli, E. y D. Tuesca. (2005). Análisis de los cambios en las comunidades de malezas en siembra directa y sus factores determinantes. Rev. de la Fac. de Agronomía, La Plata, 102 (1): 97:118.

Servicio Nacional de Sanidad y Calidad Agroalimentaria [SENASA] (2020). Disponible en: http://www.senasa.gob.ar/ casos-confirmados-de-malezas-resistentes-en-argentina

Sabattini, R. A., Sione, S. M., Ledesma, S. G., Rosenberger, L. J., Wilson, M. G., Loker, M. F., y Cinto, M. (2018). Banco de semillas de especies arbóreas en bosques nativos del espinal (Entre Ríos). Ciencia, Docencia y Tecnología Suplemento, 8(8).

Sione, S. M. J., Ledesma, S. G., Rosenberger, L. G., Galliussi, R., \& Sabattini, R. A. (2015). Soil seed bank in two successional stages of the native forest in Entre Ríos. Quebracho, (23), 62-76.

Swanton, C. J., Mahoney, K. J., Chandler, K., \& Gulden, R. H. (2008). Integrated weed management: knowledge-based weed management systems. Weed Science, 56(1), 168-172. 
Thompson, K., Bakker, J. y R. Bekker. (1997). The soil seed banks of North West Europe: methodology, density and longevity. Cambridge University Press. United Kingdom. $276 \mathrm{pp}$. 